\title{
Research on Traffic Improvement for Increasing Land Value in Tourism Region
}

\author{
Xiaojuan Hui \\ Nankai University Binhai College \\ Tianjin, China 300270
}

\author{
Weikang Liu \\ Nankai University Binhai College \\ Tianjin, China 300270
}

\begin{abstract}
Ways of improving urban land value are multiple. In recent years, the flourish of tourist industry has caused many problems. According to the increasing land demand in society, this paper proposes the research perspective of "Traffic Improvement in Tourism Region" and discusses that increasing land value in tourism region requires traffic improvement as well as corresponding design principle, basic structure and form features. From "factors influencing urban land value", this paper discusses relations between factors and starts from the branch of "traffic improvement" and emphatically analyzes the design process of improving land value in tourism regions.
\end{abstract}

\section{Keywords - traffic improvement; tourist area; land value}

\section{INTRODUCTION}

In recent years, the upgrading and reform of increasing urban traffic design changes from "vehicle-oriented" to "people-oriented", while the traffic design in tourism regions is not upgraded. Drawbacks exist in the application of traditional transportation planning to tourism regions. The huge growth of passenger flow contributes to common occurrence of problems such as difficult parking in tourism regions, traffic congestion, unclear touring routes and chaotic way of touring. They have great negative impact on the normal operation and development of tourism regions even the normal operation of cities.

This paper reflects on the traffic condition of tourism regions, upgrades transport lines and functions and improves integral level in order to improve the availability of original land and its capacity in tourist reception. Make the utmost of tourism resource and then make the original natural and human landscape and folk culture in tourism regions widely known and finally reach the goal of improving land value. In terms of design and innovation points, firstly, establish a relatively complete and comprehensive traffic system in tourism regions, including five sub-traffic systems namely static traffic system, slow-down traffic system, public transit system, road traffic system and transfer traffic system, to ameliorate current traffic problems in tourism regions and meet different tourists' requirements for travel. Secondly, apply related software technology of information visualization to traffic improvement to provide clearer and more convenient traffic information and travel experience with higher acceptability.

\section{LITERATURE REVIEW}

In traditional discipline classification, most analyses on concepts and theories of "land value" focus on the planning area. In recent years, it is gradually involved in the major of environmental art design, but it is still in the primary exploratory stage and does not form systematic theory and method basis. There are few cases researching the design about "improvement of land value" and "traffic improvement". At home, in Research on Optimizing Methods of Transportation Planning in Tourist Area, Tan Yongkai uses theoretical method of diversified integration and proposes methods of optimizing traffic planning in tourism regions.

At abroad, for example, some researches on traffic improvement and traffic designs have appeared in Boston Transportation Design Guidelines and it is significantly effective. In March 2010, the US Department of Transportation signed and issued Statement on Improvement Rules and Recommendations of Bicycle and Pedestrian from Department of Transportation to support traffic improvement.

To sum up, currently, researches on increasing tourism land value via traffic improvement mainly aim at the following two fields: one is the research on improvement of land value and the other is the research on traffic design in tourism regions, lacking the coordination of comprehensive traffic system. Therefore, the "Research on Traffic Improvement to Increase Land Value in Tourism Region" proposed in this paper carries out traffic improvement accompanied by comprehensive traffic system via a branch way of improving land value namely traffic improvement and taking tourism region as the carrier.

\section{IMPROVEMENT OF LAND VALUE IN TOURISM REGIONS}

\section{A. Traffic System in Tourism Region}

1) Current situation of system: Currently, the tourist industry in China is booming. A variety of tourist areas have flourished in China since 2015 when the yearly total number of tourists broke through 4 billion. With the huge growth of passenger flow, problems such as traffic congestion in tourist areas, single traffic form and difficult parking accompany and have greatly influenced the development of 
tourist areas and even the normal traffic circulation of relevant cities. It is mainly because of the incompatibility between traffic characteristics of tourist areas and the traditional method of transportation planning. [1]

2) Demand analysis: The functional orientation, traffic factors and traffic characteristics of tourism regions differ from that of general regions, so the analysis on its traffic demand is also different from the traditional demand analysis. [2] The idea of combining overall requirement of regions with key passenger flow and differentiating conventional trip mode from unconventional trip mode is adopted.

The unconventional trip mode in key areas is a concept relative to daily transportation in general regions. With regard to unconventional trip mode, different unconventional trip concerns and solves different problems. For instance, for relaxation on holidays, considerations must be given to comprehensive development and commercial activities, and traffic quality and traffic environment must be focused; Roads in tourism theme parks are extremely complicated and the passenger flow in parks is large, so the traffic design of these theme parks shall focus on solving problems of parking for tourists.

3) Traffic design and elements: The traffic system in tourism regions mainly includes external traffic and internal

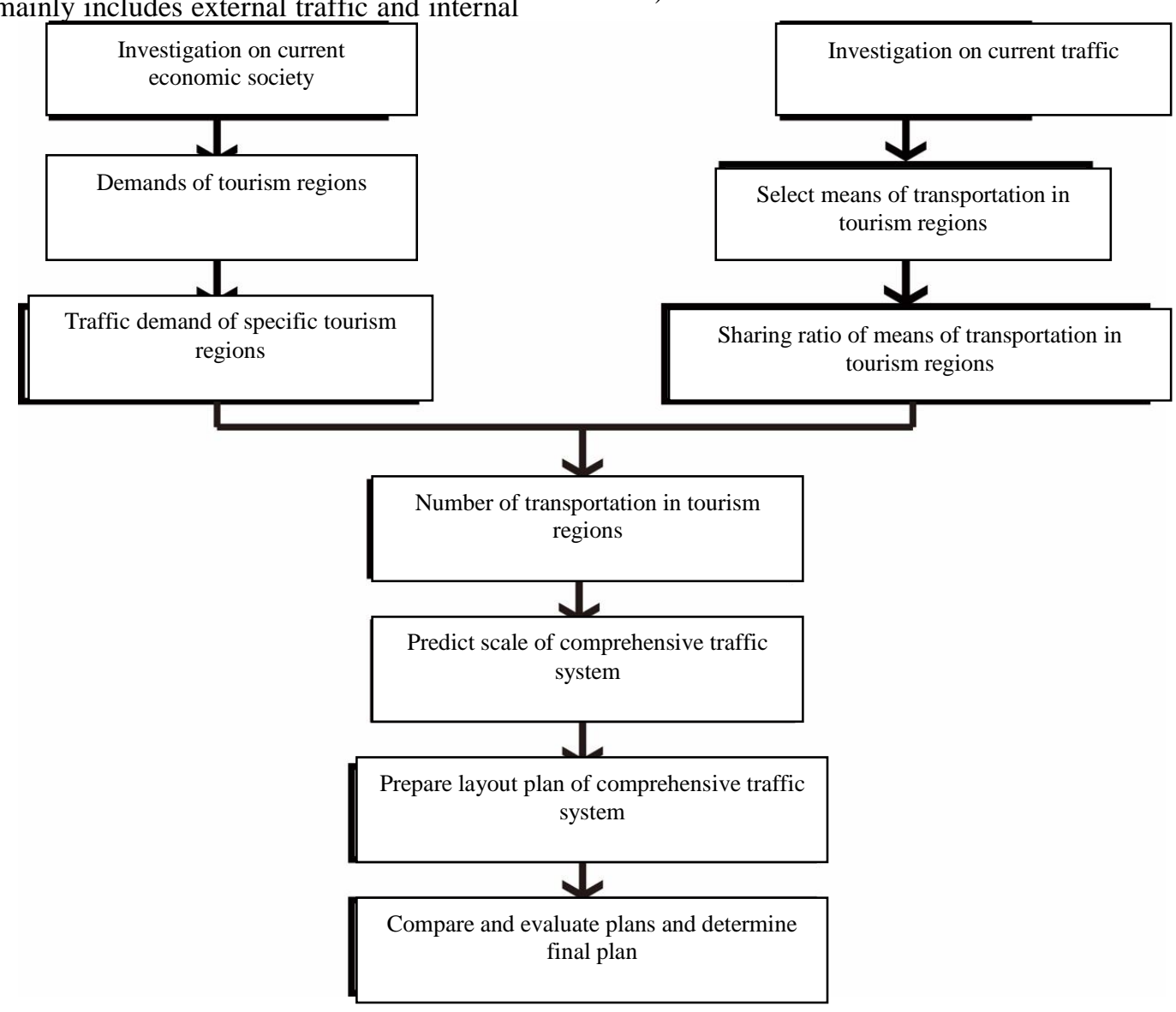

traffic. The external traffic system includes highway, aviation, railway and internal public transport of tourism regions. The internal traffic system refers to modes of transportation in tourism regions such as sightseeing vehicles, bicycles and walk. The main content of external traffic includes selection of external modes of transportation, route and supporting transport facilities. External traffic generally arranges outside or on the edge of tourism regions and should be fast and convenient. Internal traffic mainly selects modes of transportation suitable to characteristics of tourism regions and designs touring routes of modes of transportation and supporting traffic facilities. [3]

4) Design steps for layout of comprehensive traffic system: Design steps for layout of comprehensive traffic system need sufficient investigations on social economy and traffic situation. Then determine demands of tourism regions and select modes of transportation, and then the traffic demand of specific tourism regions and sharing ratio of means of transportation in different aspects and then calculate the number of transportation in tourism regions and predict the scale of comprehensive traffic system. And then prepare plans of system layout and finally compare plans and determine the best plan via evaluation (see "Fig. $1 ")$. 


\section{B. Measures of Improving Comprehensive Traffic System}

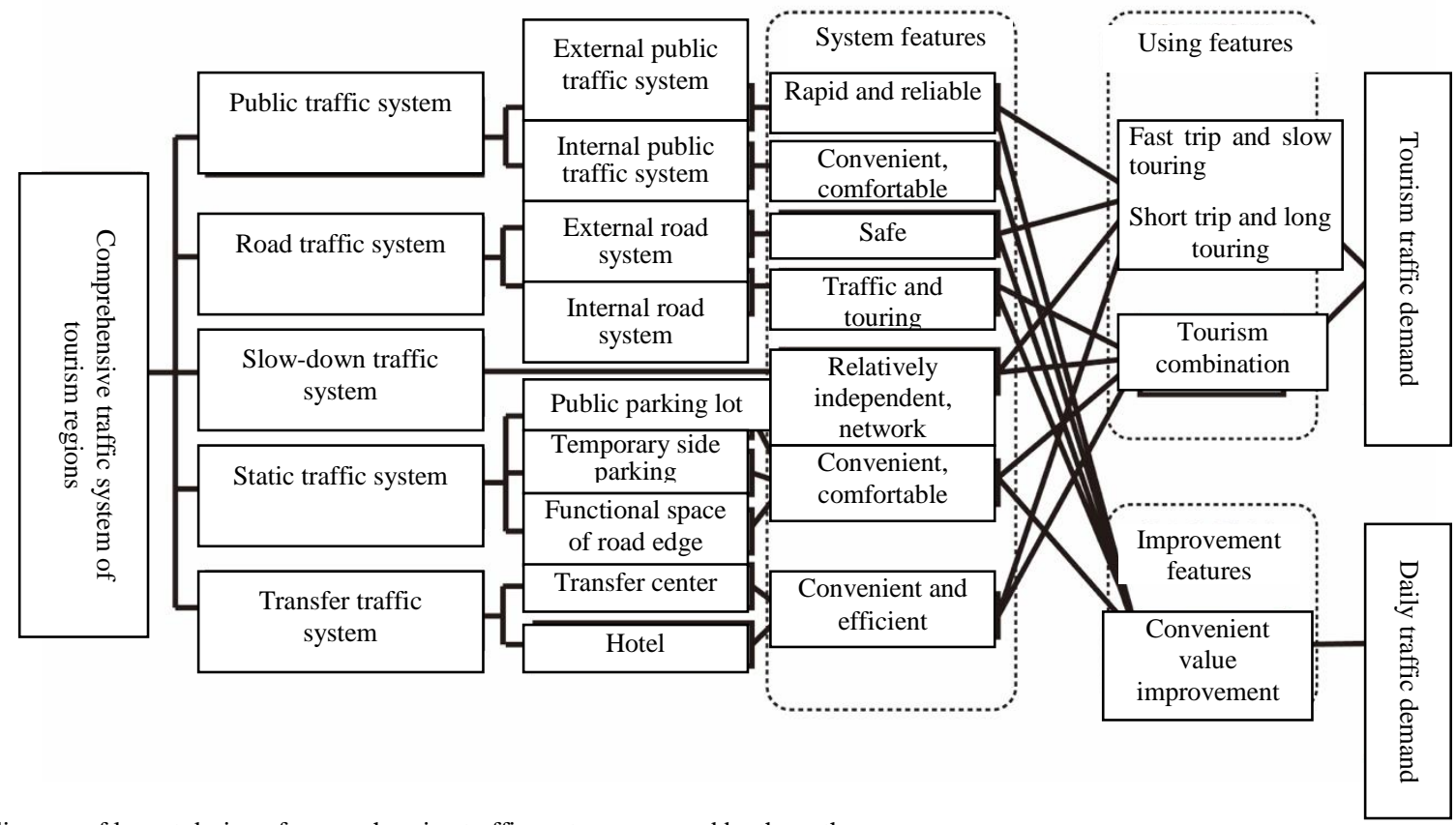

Fig. 2. Flow diagram of layout design of comprehensive traffic system, prepared by the author.

1) Goal of improvement: The goal is to meet tourists' demand and guarantee their good experience of tourism traffic via improving the traffic of tourist areas.

\section{2) Method of improvement}

a) Public traffic system: The public traffic system is divided into external public traffic system and internal public traffic system. Traffic facilities in different regions should be differentiated. Public areas adopt small blocks and focus on branch road, with artery as complement and use "multi-mode" public transport service to meet traffic demand of daily life and commuting; residential zones mainly adopt medium-sized blocks.

b) Road traffic system: Road traffic system is divided into external road traffic system and internal traffic system. The regional differentiation of facilities mainly reflects on mode of road network. It is extremely important to set pedestrian streets and visual parking space at the roadside to continuously improve tourists' environmental experience and make them have more leisure time strolling in leisure resorts. [4]

Differentiation means services provided by traffic system for different kinds of passenger flows are different. [5] For passenger flow of commuting, the external capacity of traffic system should be sufficient for convenient access, and the capacity of internal traffic system should be appropriate for proper speed; for passenger flow of large capacity and shortterm impact of exhibition tourism and characteristic tourism, the traffic system should meet the demand of convenient access and easy distribution of passenger flow; for passenger flow of leisure, a good traffic environment should be mainly provided to make them feel the sincere service attitude. [6] "Multiform" refers to the variety of alternative vehicles to adapt to local conditions. And various vehicles such as electric sightseeing vehicles, public bikes and new-type ecofriendly vehicles are adopted.

c) Slow-down traffic system: Slow-down traffic system is a relatively independent and network-based system, such as bicycles passing sidewalks. In tourism regions, it is necessary to limit the speed of tourists' vehicles and alert pedestrians; corresponding measures must be taken in non-tourism regions that are attractive for pedestrians. Static traffic management of time and space differentiation should be adopted for short-time traffic with high-capacity impact. [7]

d) Static traffic system: Static traffic system is mainly divided into three parts, public parking system, temporary roadside parking and functional space of road edge. Static traffic management of time and space differentiation should be adopted for short-time traffic with high-capacity impact. Tourists' demand for parking must be guaranteed as far as possible in rush hour and off-peak time. [8]

e) Transfer traffic system: Transfer traffic system is divided into two types, transfer centers and hotels which are common means of transfer in tourism regions. The position and convenience degree of transfer center also have great influence on traffic (see "Fig. 2").

\section{INFLUENCE OF TRAFFIC IMPROVEMENT ON INCREASING LAND VALUE OF TOURISM REGIONS}

1) Method of evaluating land value: There are many methods to evaluate land value, such as hypothetical development method, market comparison approach, land income capitalization approach, computer aided system-GIS 
application area. In fast-growing areas, hypothetical development method and urban land income capitalization approach dominate.

a) Hypothetical development method: After the completion of developing land construction project, analyze the anticipated development value of project, deduct cost, management cost, selling expenses and taxes and dues of project construction and conduct reasonable price evaluation of land use rights. The basic computational formula is: land value is equal to the value of project development minus management cost minus selling expenses minus interests minus construction cost minus taxes and dues minus relevant profit.

b) Market comparison approach: The method means comparing land to be valuated with similar lands that have transactions near the valuation date, as objective and reasonable price of appraisal object at valuation date. Lands valuated according to this method need correction of trade date, transaction, regional factor and individual factor, etc. The transaction price of sample land is corrected as the reasonable price of valuated land at valuation date. The basic computational formula is as follows: price of appraisal object is equal to correction factor of transaction date multiplying correction factor of transaction situation multiplying comparable sample price multiplying correction factor of regional factor multiplying correction factor of individual factor. The market comparison approach is applicable to the sample land with sufficient substitutability and is inapplicable to lands without transaction such as church, ancient building, school, memorial hall and temple.

c) Land income capitalization approach: To obtain future income to estimate current value. The computational formula of land income capitalization approach is as follows: $\mathrm{v}=\mathrm{ar}$, where a means net income and $\mathrm{r}$ means reduction rate.

2) Influence of traffic system on the improvement of land value: The design and improvement of comprehensive traffic system will have positive influence on tourism regions to solve corresponding traffic problems. The main effects include:

- Solving problems in rush hours of tourism regions, such as traffic congestion, single touring way and lacking commercial vitality.

- Increasing the coverage area of traffic line in tourism regions and reducing unnecessary travel time;

- Promoting the development speed of tourist industry and optimizing development prospect of tourist industry. [9]

\section{CONCLUSION}

This paper does not research the transfer system in detail. The systematic and in-depth research on transfer system and types of transfer like hotels in subsequent learning is expected, in order to make transfer system play a role in increasing land value of tourism regions. In planning of tourist areas in China, people have gradually realized traffic problems caused by ceaseless reinforcement of motor vehicle traffic and are changing values of planning and design. Compared with other design approaches, ideas and way of thinking of improvement in "traffic improvement" can be better followed by tourism regions in China. Traffic improvement concerns people-oriented society and future development and determines the trend of group behaviors in cities. Topics of traffic system research attract attentions from an increasing number of scholars, designers and social institutions that devote wisdom and energy to this field. It is because the seemingly ordinary traffic improvement affects and organizes people's life and vitality reshaping and overall travel efficiency of tourist areas. A better life will be created through designing better traffic systems used by us.

\section{REFERENCES}

[1] Wu Guoying. Ways of Effectively Improving Urban Land Value, Guangzhou Panyu Institute of Urban Architecture Design, 223-224. (in Chinese)

[2] Zhang Lin, Li Ying. Review on Methods and Practice of Land Assessment of Foreign Brownfield Site [J], Faculty of Managemen and Economics, Dalian University of Technology, 2012, 204-205. (in Chinese)

[3] Zhong Shien, Zhang Jie, Han Guosheng, et al. Basic Theory of Space Pattern of Tourist Flow: Problem Analysis and Its Prospect [J], Human Geography, 2010, 31-36. (in Chinese)

[4] Mings R, K McHugh, 1992 The spatial configuration of travel to Yellowstone National Park [J].Journal of Travel Research, 30 30: 3846. (in Chinese)

[5] Tan Yongkai, Research on Optimizing Methods of Transportation Planning in Tourist Area on the Basis of Diversified Integration, Shanghai Urban Construction Design and Research Institute (Group) Co., Ltd. Shanghai, 200125, 25-26. (in Chinese)

[6] Bao Jigang, Zheng Haiyan. Evolution of Space Structure of Domestic Tourist Market in Guilin [J], Journal of Geographical Sciences, 2002, 5757(1): 196-206. (in Chinese)

[7] Yang Huiwei, Experience Summary of Complete Street Theory and Practice in Boston and Enlightenment on Our Country's Street Design [J], School of Architecture and Urban Planning, Beijing University of Civil Engineering and Architecture, 214-215. (in Chinese)

[8] Hu Hao. Analysis on Space Distribution of China's Excellent Tourism Cities and Regional Disparity of Transport Availability [J/OL], Geographical Science, 2013, 15-16. (in Chinese)

[9] Lu Song. Research Progress and Enlightenment of Tourist Traffic [J], Tropical Geography, 2009,394-399. (in Chinese) 